\title{
Assessing Muscular Oxygenation During Incremental Exercise Using Near-Infrared Spectroscopy: Comparison of Three Different Methods
}

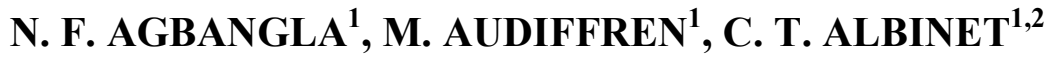 \\ ${ }^{1}$ Centre de Recherches sur la Cognition et l'Apprentissage (UMR7295), Université de Poitiers and \\ Université François-Rabelais de Tours, Poitiers, France, ${ }^{2}$ Laboratoire Sciences de la Cognition, \\ Technologie, Ergonomie (SCoTE), Université de Toulouse, INU Champollion, Albi, France
}

Received February 7, 2017

Accepted May 2, 2017

On-line September 22, 2017

\section{Summary}

Using continuous-wave near-infrared spectroscopy (NIRS), this study compared three different methods, namely the slope method (SM), the amplitude method (AM), and the area under the curve (AUC) method to determine the variations of intramuscular oxygenation level as a function of workload. Ten right-handed subjects ( $22 \pm 4$ years) performed one isometric contraction at each of three different workloads (30\%, $50 \%$ and $90 \%$ of maximal voluntary strength) during a period of twenty seconds. Changes in oxyhemoglobin $\left(\Delta\left[\mathrm{HbO}_{2}\right]\right)$ and deoxyhemoglobin $(\Delta[\mathrm{HHb}])$ concentrations in the superficial flexor of fingers were recorded using continuous-wave NIRS. The results showed a strong consistency between the three methods, with standardized Cronbach alphas of 0.87 for $\Delta[\mathrm{HHb}]$ and 0.95 for $\Delta\left[\mathrm{HbO}_{2}\right]$. No significant differences between the three methods were observed concerning $\Delta[\mathrm{HHb}]$ as a function of workload. However, only the SM showed sufficient sensitivity to detect a significant decrease in $\Delta\left[\mathrm{HbO}_{2}\right]$ between $30 \%$ and $50 \%$ of workload $(p<0.01)$. Among these three methods, the SM appeared to be the only method that was well adapted and sensitive enough to determine slight changes in $\Delta\left[\mathrm{HbO}_{2}\right]$. Theoretical and methodological implications of these results are discussed.

\section{Key words}

NIRS • Muscle hemodynamics • Linear slope • Amplitude • Area under the curve

\section{Corresponding author}

C. T. Albinet, SCoTE, INU Champollion, Place Verdun, 81000 Albi, France. E-mail: cedric.albinet@univ-jfc.fr

\section{Introduction}

Since the end of the 1980s, continuous-wave near-infrared spectroscopy (NIRS) has been increasingly used as a noninvasive technique for investigating local muscle oxygenation changes during exercise or rest (Ferrari et al. 1997, Ferrari et al. 2011). The validity of NIRS has been examined by different studies (Sako et al. 2001, Van Beekvelt et al. 2001), and the results obtained using this technique are in agreement with those obtained by other imaging techniques such as phosphorus magnetic resonance spectroscopy (P-MRS) (Sako et al. 2001) and blood gas analysis (Van Beekvelt et al. 2001). Several studies have also examined the reproducibility of this optical imaging technique (Van Beekvelt et al. 2002, Celie et al. 2012, Lacroix et al. 2012). In this way, agreement was observed between measurements of oxygen consumption of the superficial flexor of fingers performed on three different days (Van Beekvelt et al. 2002). Based on intra-class correlation coefficients (ICC), a high reproducibility of data derived from NIRS was shown (ICC $=0.85-1$, Lacroix et al. 2012). Furthermore, Celie et al. (2012) also confirmed the reproducibility of this optical technique, particularly when the intensity of the muscular contraction was increased. Beside its reproducibility, there has been shown a strong relationship between NIRS signals and electromyography data during static and sinusoidal isometric exercises of the biceps brachii muscle (Felici et al. 2009). However, few studies have specifically examined the sensitivity of NIRS to measure muscle oxygen consumption as 
a function of workload.

At the muscle level, near-infrared light is absorbed by hemoglobin and myoglobin (Ferrari et al. 2011). Because these two chromophores have identical spectral characteristics, it is impossible to distinguish their respective light absorption (Binzoni et al. 1999, Van Beekvelt et al. 2001, Bhambhani 2004). As the investigation of muscular oxidative metabolism is independent of the oxygen source, hemoglobin is then the term used to name the two chromophores (Lacroix et al. 2012). In general, the main recorded parameters using NIRS to study muscular oxidative metabolism are the following: 1) changes in oxyhemoglobin concentrations $\left(\Delta\left[\mathrm{HbO}_{2}\right]\right)$ and deoxyhemoglobin concentrations $(\Delta[\mathrm{HHb}]) ; 2)$ changes in total hemoglobin concentrations $(\Delta[\mathrm{HbT}])$; and 3$)$ muscle oxygen saturation $\left(\mathrm{SmO}_{2}\right)$ (Ferrari et al. 2011).

To determine the level of change in hemodynamic parameters, previous studies have used different methods such as the slope method (SM) (Quaresima et al. 2001), the amplitude method (AM) (Celie et al. 2012) and the area under the curve (AUC) method (Manfredini et al. 2009). The SM consists of calculating, in the entire recorded signals window, a linear regression to obtain the slope coefficient, which indicates the magnitude and direction of the hemodynamic parameters (e.g. $\Delta\left[\mathrm{HbO}_{2}\right], \Delta[\mathrm{HHb}]$ ). The AM consists of subtracting the level obtained during a resting state (typically the last $10 \mathrm{~s}$ of the rest period) from an activation period (typically the last $10 \mathrm{~s}$ of the contraction) after reaching a plateau for each trial. To determine the amplitude of changes of the hemodynamic parameters, the AUC method integrates the surface obtained under the curve of the hemodynamic parameter changes during the entire recorded signal window.

As stated above, few studies have used NIRS to investigate muscle oxygen consumption during active contraction, and those studies have used different quantification methods (Quaresima et al. 2001, Celie et al. 2012, Manfredini et al. 2009). Among these studies, only Celie et al. (2012) used different workloads in their protocol. Their results indicate that the muscle's hemodynamic response (increase in $\Delta[\mathrm{HHb}]$ and decrease in $\left.\Delta\left[\mathrm{HbO}_{2}\right]\right)$ appears somewhat proportional to workload. However, these authors did not specifically address this issue in their study. To our knowledge, no study has yet examined the concurrent validity of these three methods (SM, AM, AUC) or examined whether they show the same sensitivity to changes in workload. Accordingly, the aim of the present study was to examine, in the same protocol involving the same participants, the sensitivity of these three methods to determine slight changes in hemodynamic parameters during an incremental isometric handgrip exercise.

\section{Material and Methods}

\section{Participants}

Ten right-handed, healthy Caucasian adults ( 5 men and 5 women) participated in our study. Their mean \pm SD age and body mass index (BMI) were $22 \pm 4$ years and $21.11 \pm 2.4 \mathrm{~kg} / \mathrm{m}^{2}$, respectively. Because of potential effects of subcutaneous fat on NIRS signals (McCully and Hamaoka 2000), we recruited non-obese subjects whose BMI was no more than $25 \mathrm{~kg} / \mathrm{m}^{2}$ (range $17-24 \mathrm{~kg} / \mathrm{m}^{2}$ ). Indeed, subcutaneous fat greatly influences the NIRS signal intensity which must pass through the muscle (Hamaoka et al. 2011). All the participants gave their written informed consent to participate in the study, which complied with the declaration of Helsinki for human experimentation.

\section{Experimental design}

Handgrip strength was measured using a digital strain-gauge dynamometer (Takei TK 200, Takei Scientific Instruments, Tokyo, Japan) with an accuracy of $\pm 2 \mathrm{~kg}$. To standardize the muscle location for all participants, the distance (D) between the medial epicondylus humerus and the processus coronoideus ulnae was measured. The transmitter optode was then positioned at a distance equal to $1 / 3 \mathrm{D}$ from the medial epicondylus. The receiving optode was positioned laterally, $4 \mathrm{~cm}$ from the transmitter optode, allowing measurement of muscle oxygenation of the flexor digitorum superficialis. The participants were seated in front of a table with their non-dominant upper limb along the body and their right hand in supination so that their forearm formed an angle of approximately $130^{\circ}$ with their arm. The width of the handle was adjusted to the size of the hand to ensure that the middle phalanx rested on the inner handle. The participants were allowed to perform one test trial. Then, after a complete 30 -s period of rest, the participants performed one isometric contraction at each of three different workloads: $30 \%$, $50 \%$ and $90 \%$ of maximal voluntary strength (MVS). Isometric contraction was chosen because this form of contraction is more prevalent in the studies (Quaresima et al. 2001, Muthalib et al. 2010, Celie et al. 2012) and 
minimizes noise due to movements. The duration of each isometric contraction was $20 \mathrm{~s}$ with a rest period equal to $60 \mathrm{~s}$ between each contraction.

A continuous-wave NIRS (Oxymon Mk III, Artinis Medical Systems, Zetten, The Netherlands) was used to measure the changes in concentration of oxygenated hemoglobin $\left(\Delta\left[\mathrm{HbO}_{2}\right]\right)$, deoxygenated hemoglobin $(\Delta[\mathrm{HHb}])$ and total hemoglobin $(\Delta[\mathrm{HbT}])$, with a sampling rate set at $10 \mathrm{~Hz}$ (Quaresima et al. 2001, Muthalib et al. 2010). This device measures only hemodynamic relative changes and may be relatively sensitive to movement artifacts (Wolf et al. 2007). This constraint was limited by using isometric contractions. The measurement of changes in concentration involves the determination of optical densities of two wavelengths ( $857 \mathrm{~nm}$ and $764 \mathrm{~nm}$ ) in the near-infrared range once they have passed through the muscle. The optical density of these two wavelengths, which are absorbed by oxygenated hemoglobin $\left(\mathrm{HbO}_{2}\right)$ and deoxygenated hemoglobin $(\mathrm{HHb})$, respectively, are converted to concentrations of oxygenated hemoglobin $\left[\mathrm{HbO}_{2}\right]$ and deoxygenated hemoglobin [HHb] by the modified BeerLambert law (Villringer and Chance 1997, see Eq. [1]):

$$
\mathrm{A}=\varepsilon \times \mathrm{c} \times \mathrm{d} \times \mathrm{DPF}+\mathrm{G}
$$

where $\mathrm{A}$ is the absorption of light expressed as optical density, $\varepsilon$ is a specific extinction coefficient, $\mathrm{c}$ is the chromophore concentration, $\mathrm{d}$ is the traveled distance, DPF is the differential path length factor and $G$ is the loss of signal due to light scattering.

\section{Processing of NIRS data}

NIRS data were analyzed using commercially available software (Oxysoft, Artinis Medical Systems, Zetten, The Netherlands). A low-pass filter with a cutoff frequency of $0.7 \mathrm{~Hz}$ was used to remove the heart rate signal. Then, the changes in hemodynamic parameters at each workload $(30 \%, 50 \%$, and $90 \%$ of MVS) were calculated using the SM, AM and AUC methods. Concerning the SM, a linear regression technique was applied to the 20-s time-series data to obtain the slope coefficient. For the AM, we subtracted the level obtained at the resting state (mean of the last $10 \mathrm{~s}$ of the rest period) from the one of the activation period (mean of the last $10 \mathrm{~s}$ of the isometric contraction). Finally, for the AUC, we determined the area under the curve of each hemodynamic parameter for the 20-s isometric contraction.

Statistical analysis

Statistical analyses were performed using STATISTICA software version 7. The assumption of data normality and homogeneity of distributions was assessed using Kolmogorov-Smirnov and Levene tests, respectively. A standardized Cronbach alpha was computed to measure the consistency between the three methods. To examine the sensitivity of each method as a function of workload, separate ANOVAs with 3 repeated measures (30\% vs. $50 \%$ vs. $90 \%$ ) on $\Delta\left[\mathrm{HbO}_{2}\right]$ and $\Delta[\mathrm{HHb}]$ were first conducted. Second, to compare the three different methods, all $\Delta\left[\mathrm{HbO}_{2}\right]$ and $\Delta[\mathrm{HHb}]$ raw data were transformed into $z$-scores (using means and standard deviations). Separate 3 (workload: $30 \%$ vs. $50 \%$ vs. $90 \%$ ) $\times 3$ (method: SM vs. AM vs. AUC) MANOVAs with repeated measures were performed on the z-scores of $\Delta\left[\mathrm{HbO}_{2}\right]$ and $\Delta[\mathrm{HHb}]$ data. For significant results, post hoc mean comparisons were performed using Bonferroni corrections for multiple comparisons. The level of significance was set at $\mathrm{p}<0.05$ and partial estimated effect sizes $\left(\eta_{p}^{2}\right)$ were reported for significant results.

\section{Results}

Figure 1 shows an illustration of the typical hemodynamic changes observed in one participant; $\Delta[\mathrm{HHb}]$ increased and $\Delta\left[\mathrm{HbO}_{2}\right]$ decreased as a function of workload. Globally, the standardized Cronbach alphas were 0.87 for $\Delta[\mathrm{HHb}]$ and 0.95 for $\Delta\left[\mathrm{HbO}_{2}\right]$, indicating a strong consistency between the three methods. The bivariate correlation coefficients between the three methods at each workload were all significant (between 0.66 and 0.98) with the exception of the AUC method and the $\mathrm{SM}$ at $30 \%$ of workload for both $\Delta\left[\mathrm{HbO}_{2}\right]$ and $\Delta[\mathrm{HHb}]$ (all $\mathrm{r}=0.5$, ns) and the AUC method and the $\mathrm{AM}$ for $\Delta[\mathrm{HHb}]$ at $30 \%$ of $\operatorname{workload}(\mathrm{r}=0.57, \mathrm{~ns})$.

\section{Slope method}

The analyses showed a main effect of workload on $\Delta\left[\mathrm{HbO}_{2}\right]$ and on $\Delta[\mathrm{HHb}]: \mathrm{F}(2,18)=15.8 ; \mathrm{p}<0.01$; $\eta_{p}^{2}=0.63 \quad$ and $\quad \mathrm{F}(2,18)=20.12 ; \quad \mathrm{p}<0.01 ; \quad \eta_{p}^{2}=0.69$, respectively. Post hoc analyses showed that there was a significant difference between each workload for both $\Delta\left[\mathrm{HbO}_{2}\right]$ and $\Delta[\mathrm{HHb}]$ slope coefficients (Fig. 2). 


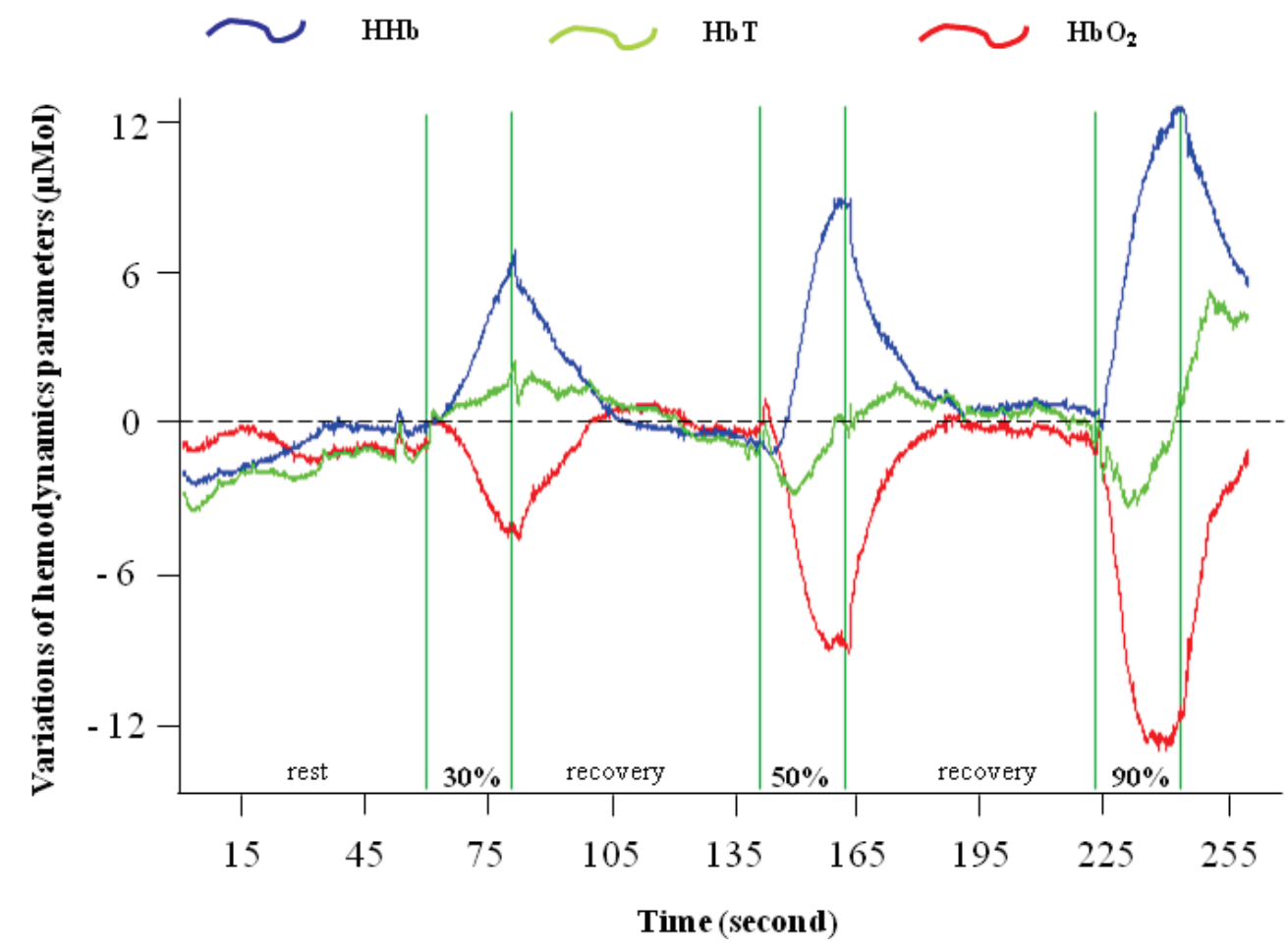

Fig. 1. Illustration of the typical variations of hemodynamic parameters as a function of workload for one participant.

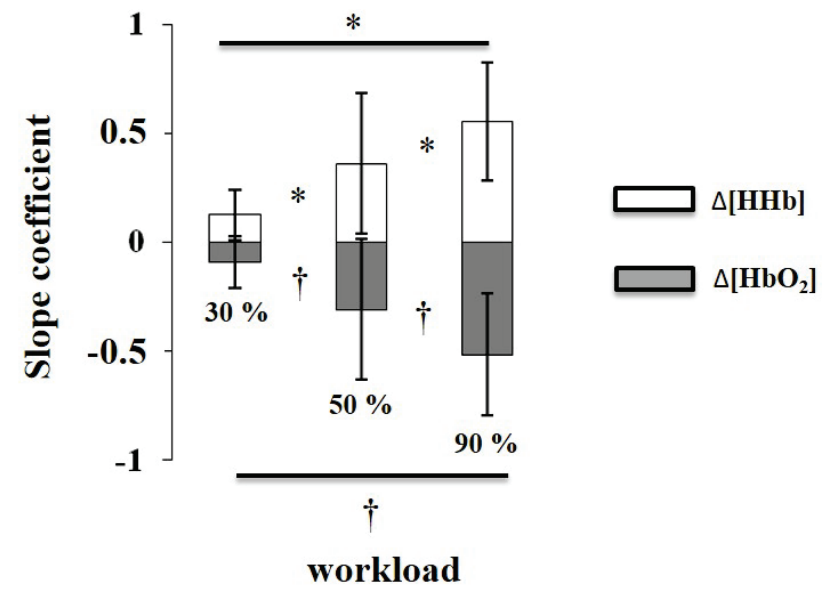

Fig. 2. Mean results using the slope method as a function of workload. Bars represent standard deviation. * $\mathrm{p}<0.05$ for $\mathrm{HHb}$ and $+\mathrm{p}<0.05$ for $\mathrm{HbO}_{2}$.

\section{Amplitude method}

The analyses showed a main effect of workload on $\Delta\left[\mathrm{HbO}_{2}\right]$ and on $\Delta[\mathrm{HHb}]: \mathrm{F}(2,18)=14.51 ; \mathrm{p}<0.01$;

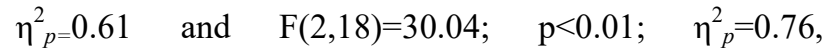
respectively. For $\Delta\left[\mathrm{HbO}_{2}\right]$ data, post hoc analyses showed that there was a significant difference between $30 \%$ and $90 \%$ and between $50 \%$ and $90 \%$, but not between $30 \%$ and $50 \%(\mathrm{p}=0.1$; Fig. 3$)$. For $\Delta[\mathrm{HHb}]$ data, post hoc analyses showed that there was a significant difference between each workload.

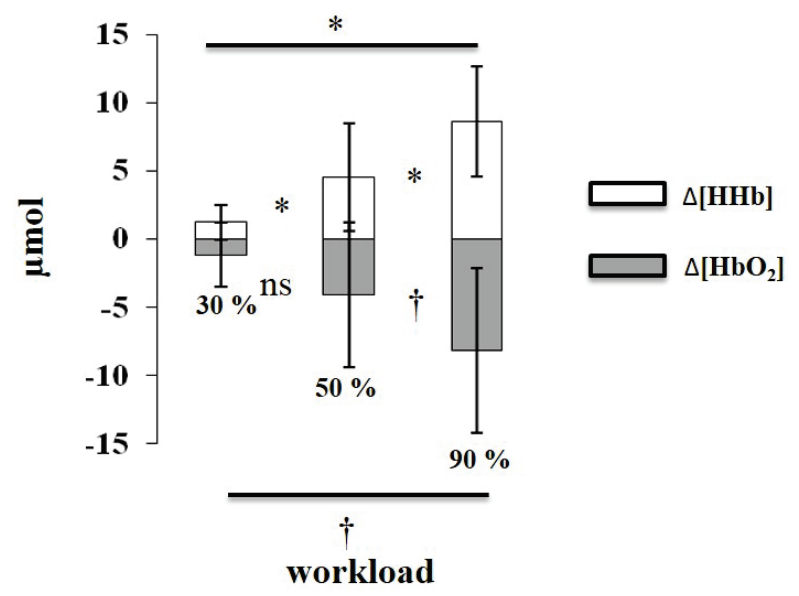

Fig. 3. Mean results using the amplitude method as a function of workload. Bars represent standard deviation. $* \mathrm{p}<0.05$ for $\mathrm{HHb}$ and $t \mathrm{p}<0.05$ for $\mathrm{HbO}_{2}$.

Area under the curve method

The analyses showed a main effect of workload on $\Delta\left[\mathrm{HbO}_{2}\right]$ and on $\Delta[\mathrm{HHb}]: \mathrm{F}(2,18)=10.73 ; \mathrm{p}<0.01$; $\eta_{p}^{2}=0.54 \quad$ and $\quad \mathrm{F}(2,18)=32.48 ; \quad \mathrm{p}<0.01 ; \quad \eta_{p}^{2}=0.78$, respectively. For $\Delta\left[\mathrm{HbO}_{2}\right]$ data, post hoc analyses showed that there was only a significant difference between $30 \%$ and $90 \%$ (Fig. 4). For $\Delta[\mathrm{HHb}]$ data, post hoc analyses showed that there was a significant difference between each workload. 


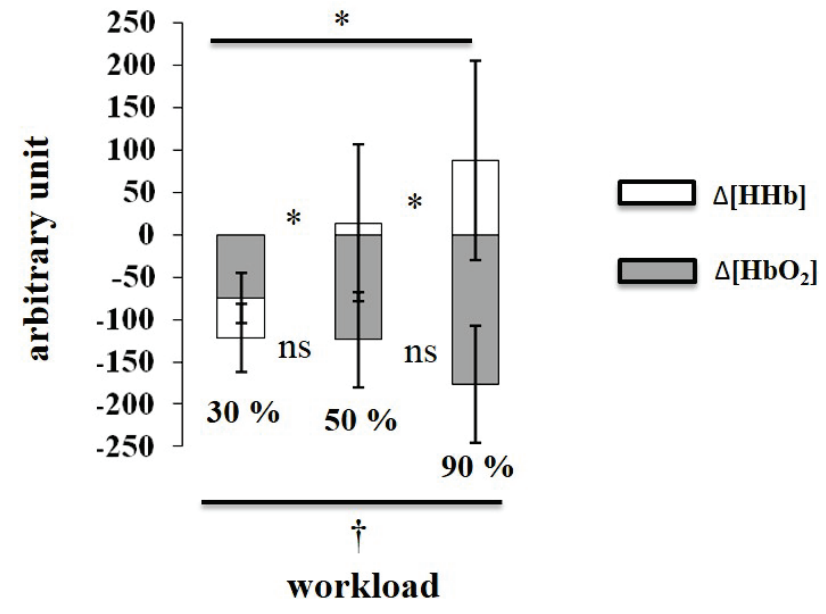

Fig. 4. Mean results using the area under the curve method as a function of workload. Bars represent standard deviation. $* p<0.05$ for $\mathrm{HHb}$ and $+\mathrm{p}<0.05$ for $\mathrm{HbO}_{2}$.

\section{Comparison of the three methods}

The MANOVA performed on $\Delta\left[\mathrm{HbO}_{2}\right] z$-scores showed a significant effect of workload and a main effect of the method: Wilk's lambda $=0.22 ; \mathrm{F}(2,9)=15.85$; $\mathrm{p}=0.001$ and Wilk's lambda $=0.32 ; \mathrm{F}(2,9)=9.53 ; \mathrm{p}=0.005$, respectively. A significant workload $\times$ method interaction was also revealed: Wilk's lambda $=0.12 ; \mathrm{F}(4,7)=11.73$; $\mathrm{p}=0.003$. Post hoc analyses showed that only the SM was sufficiently sensitive to indicate a significant difference between $30 \%$ and $50 \%$ workloads $(\mathrm{p}<0.01)$.

For $\Delta[\mathrm{HHb}] \quad z$-scores, a significant main effect of workload was revealed: Wilk's lambda $=0.17$; $F(2,9)=21.43 ; p=0.0003$. The main effect of the method was not significant $(\mathrm{p}=0.22)$.

\section{Discussion}

In the present study, NIRS was used to examine relative changes in muscle oxygenation parameters as a function of three workloads during an isometric exercise. These muscle oxygenation changes were calculated using three different methods that are often reported in the literature to investigate their respective sensitivity for determining the magnitude of hemodynamic parameters as a function of workload.

In regard to the effect of workload, the results showed a consistent decrease in $\Delta\left[\mathrm{HbO}_{2}\right]$ and an increase in $\Delta[\mathrm{HHb}]$ induced by the isometric contractions as a function of workload (Fig. 1). This result is important because no study has specifically examined the sensitivity of the hemodynamic parameters as a function of workload.
This evolution of the hemodynamic parameters during muscular contraction can be explained by an important and local consumption of oxygen. For example, it was shown that at $10 \%$ of maximum voluntary contraction, the oxygen consumption of the superficial flexor and brachioradial is five times higher than the quantity of oxygen needed at rest (Van Beekvelt et al. 2001). Recent studies also confirmed the increase in oxygen consumption during a voluntary isometric contraction (Ryan et al. 2012, Ryan et al. 2013). These changes in oxygenation concentration would represent oxygen needed by the muscle mitochondria during the contraction. Van Beekvelt et al. (2002) showed that local muscle oxygen consumption at rest, as well as during exercise, can be reliably measured by NIRS. Our results confirm this reliability of the continuous-wave NIRS technique.

The comparison of the sensitivity to workload between the three different methods used in the present study showed that only the SM provided a significant decrease of $\Delta\left[\mathrm{HbO}_{2}\right]$ concentration between $30 \%$ and $50 \%$ of MVS. The AM and the AUC method only detected significant variations between $30 \%$ and $90 \%$ and between $50 \%$ and $90 \%$ of MVS. However, the three methods appeared to be equivalent in detecting variations in $[\mathrm{HHb}]$ as a function of the three manipulated workloads. During an isometric contraction with forces varying between $12 \%, 18 \%$ and $24 \%$ of the maximum voluntary isometric contraction, Sako et al. (2001) calculated the changes in $\left[\mathrm{HbO}_{2}\right]$ using the least-square method and showed important and significant decreases. The diminution in $\left[\mathrm{HbO}_{2}\right]$ was strongly correlated $(\mathrm{r}=0.97)$ with the decrease in phosphocreatine (Pcr), measured with P-MRS (Sako et al. 2001). This result shows that a slight increase of workload contraction can induce an important variation of hemodynamic parameters. Using the SM, the variation of workload contraction between $30 \%$ and $50 \%$ induces important changes in hemodynamic parameters. Therefore, it is important to use a method that is sufficiently sensitive to detect slight changes, particularly in the case of a small sample of participants. In our study, only the SM method was sufficiently sensitive to detect the changes between $30 \%$ and $50 \%$ of MVS. Globally, using the SM, $\Delta[\mathrm{HHb}]$ increased by $23 \%, 20 \%$ and $43 \%$ between $30 \%, 50 \%$ and $90 \%$ of MVS, respectively, and $\Delta\left[\mathrm{HbO}_{2}\right]$ decreased by $22 \%, 21 \%$ and $42 \%$ between $30 \%, 50 \%$ and $90 \%$ of MVS, respectively.

In the present study, the SM was shown to be the 
most sensitive method for detecting significant muscular $\left[\mathrm{HbO}_{2}\right]$ decreases at low levels of workload (between $30 \%$ and $50 \%$ of MVS) and for a small sample of participants. The sensitivity of this method could be explained by the characteristics of the $\left[\mathrm{HbO}_{2}\right]$ signal, which increases linearly during the entire activation period (Fig. 1) and thus appears particularly appropriate for this type of method. However, the basis of the AM, which computes the mean difference between rest and contraction periods during $10 \mathrm{~s}$ windows, is that only a portion of the entire signal, thought to be representative of the complete hemodynamics, is captured. This method seems particularly appropriate when the signal reaches and maintains a plateau, which was clearly not the case for the hemodynamic data in the present study. As such, this could indicate that this method is not well adapted for detecting significant $\left[\mathrm{HbO}_{2}\right]$ decreases as a function of slight changes in low levels of MVS. The AUC method, similar to the SM, uses the entire activation period for computing hemodynamic changes but was shown in our study to be the less sensitive method for detecting $\left[\mathrm{HbO}_{2}\right]$ changes. This result deserves future studies to more specifically examine this lack of sensitivity. Taken together, the results of this study favor the use of the SM, which is more sensitive and well adapted to this type of signal because NIRS signals have a linear evolution in most investigations of muscle oxygenation (Ferrari et al. 1997). Moreover, the SM is easy to use because it requires only a linear regression on the NIRS signals.

Some potential limitations of the present study should be addressed. First, quantifying intramuscular oxygenation changes with NIRS requires the occlusion technique to control the circulation (Van Beekvelt et al.
2001). This technique estimates the microcirculation status of muscle (Gerovasili et al. 2010). In our study, we did not use this method. However, this method is used to precisely calculate $\mathrm{mVO}_{2}$ (oxygen consumption of muscle). In our study, this index was not the index of interest because the parameters that we have examined were $\Delta\left[\mathrm{HbO}_{2}\right]$ and $\Delta[\mathrm{HHb}]$. Second, we did not counterbalance the three workloads during the experiment. Although this could be a clear limitation to ascertain that muscle oxygenation changes were actually due to workload increases and not due to time on task or fatigue, this non-counterbalancing had no effect on the estimation of the sensitivity of the three methods, which was the principal aim of the study. Finally, although sufficiently powered to detect significant differences, our study's sample size was quite small and a replication of these results using a larger population with a direct measure of fat thickness would strengthen the conclusion.

In conclusion, the important difference between the three methods (SM, AM, AUC) used in this investigation is their sensitivity in the quantification of variations in muscle $\left[\mathrm{HbO}_{2}\right]$ according to workload. The SM appears to be a well-adapted, user-friendly method to determine slight changes in hemodynamic parameters.

\section{Conflict of Interest}

There is no conflict of interest.

\section{Acknowledgements}

This work was supported by grants from the Regional Council of Poitou-Charentes and the European Funds for Regional Development (FeDER).

\section{References}

BHAMBHANI YN: Muscle oxygenation trends during dynamic exercise measured by near-infrared spectroscopy. Can J Appl Physiol 29: 504-523, 2004.

BINZONI T, COLIER W, HILTBRAND E, HOOFD L, CERRETELLI P: Muscle O(2) consumption by NIRS: a theoretical model. J Appl Physiol 87: 683-688, 1999.

CELIE B, BOONE J, VAN COSTER R, BOURGOIS J: Reliability of near infrared spectroscopy (NIRS) for measuring forearm oxygenation during incremental handgrip exercise. Eur J Appl Physiol 112: 2364-2374, 2012.

FELICI F, QUARESIMA V, FATTORINI L, SBRICCOLI P, FILLIGOI GC, FERRARI M: Biceps brachii myoelectric and oxygenation changes during static and sinusoidal isometric exercises. J Electromyogr Kinesiol 19: e1-e11, 2009.

FERRARI M, BINZONI T, QUARESIMA V: Oxidative metabolism in muscle. Philos Trans R Soc Lond B Biol Sci 352: 677-683, 1997.

FERRARI M, MUTHALIB M, QUARESIMA V: The use of near-infrared spectroscopy in understanding skeletal muscle physiology: recent developments. Philos Trans A Math Phys Eng Sci 369: 4577-4590, 2011. 
GEROVASILI V, DIMOPOULOS S, TZANIS G, ANASTASIOU-NANA M, NANAS S: Utilizing the vascular occlusion technique with NIRS technology. Int J Ind Ergon 40: 218-222, 2010.

HAMAOKA T, MCCULLY KK, NIWAYAMA M, CHANCE B: The use of muscle near-infrared spectroscopy in sport, health and medical sciences: recent developments. Philos Trans A Math Phys Eng Sci 369: 4591-4604, 2011.

LACROIX S, GAYDA M, GREMEAUX V, JUNEAU M, TARDIF JC, NIGAM A: Reproducibility of near-infrared spectroscopy parameters measured during brachial artery occlusion and reactive hyperemia in healthy men. J Biomed Opt 17: 077010, 2012.

MANFREDINI F, MALAGONI AM, FELISATTI M, MANDINI S, MASCOLI F, MANFREDINI R, BASAGLIA N, ZAMBONI P: A dynamic objective evaluation of peripheral arterial disease by near-infrared spectroscopy. Eur J Vasc Endovasc Surg 38: 441-448, 2009.

MCCULLY KK, HAMAOKA T: Near-infrared spectroscopy: what can it tell us about oxygen saturation in skeletal muscle? Exerc Sport Sci Rev 28: 123-127, 2000.

MUTHALIB M, JUBEAU M, MILLET GY, MAFFIULETTI NA, FERRARI M, NOSAKA K: Biceps brachii muscle oxygenation in electrical muscle stimulation. Clin Physiol Funct Imaging 30: 360-368, 2010.

QUARESIMA V, COLIER WN, VAN DER SLUIJS M, FERRARI M: Nonuniform quadriceps O2 consumption revealed by near infrared multipoint measurements. Biochem Biophys Res Commun 285: 1034-1039, 2001.

RYAN TE, ERICKSON ML, BRIZENDINE JT, YOUNG HJ, MCCULLY KK: Noninvasive evaluation of skeletal muscle mitochondrial capacity with near-infrared spectroscopy: correcting for blood volume changes. $J$ Appl Physiol 113: 175-183, 2012.

RYAN TE, BRIZENDINE JT, MCCULLY KK: A comparison of exercise type and intensity on the noninvasive assessment of skeletal muscle mitochondrial function using near-infrared spectroscopy. $J$ Appl Physiol 114: 230-237, 2013.

SAKO T, HAMAOKA T, HIGUCHI H, KUROSAWA Y, KATSUMURA T: Validity of NIR spectroscopy for quantitatively measuring muscle oxidative metabolic rate in exercise. J Appl Physiol 90: 338-344, 2001.

VAN BEEKVELT MC, COLIER WN, WEVERS RA, VAN ENGELEN BG: Performance of near-infrared spectroscopy in measuring local $\mathrm{O} 2$ consumption and blood flow in skeletal muscle. $J$ Appl Physiol 90: 511-519, 2001.

VAN BEEKVELT MC, VAN ENGELEN BG, WEVERS RA, COLIER WN: In vivo quantitative near-infrared spectroscopy in skeletal muscle during incremental isometric handgrip exercise. Clin Physiol Funct Imaging 22: 210-217, 2002.

VILLRINGER A, CHANCE B: Non-invasive optical spectroscopy and imaging of human brain function. Trends Neurosci 20: 435-442, 1997.

WOLF M, FERRARI M, QUARESIMA V: Progress of near-infrared spectroscopy and topography for brain and muscle clinical applications. J Biomed Opt 12: 062104, 2007. 